\title{
Cervical consistency index is a valid predictor of preterm birth in low-risk pregnant women
}

\author{
Parichehr Pooransari*, Fatemeh Salehi*, Maryam Afrakhteh**, \\ Masoumeh Mirzamoradi**, Soraya Saleh Gargari**, and Omid Ghatreh Samani***
}

\section{ABSTRACT}

\section{BACKGROUND}

Preterm birth (PTB) remains a global problem associated with perinatal morbidity, including low birth weight, growth retardation and irreversible damage to the nervous system. The objective of this study was to determine the predictive value of cervical consistency index (CCI) to indicate the occurrence of PTB in mid trimester screening of low risk pregnant women.

\section{METHODS}

This was a prospective study conducted on low-risk pregnant women at 14-28 weeks of pregnancy. The cervical length (CL) and AP cervical diameter were measured and the CCI determined according to the formula AP2/ AP $1 * 100$. The ROC curves were drawn according to gestational age and the sensitivity and the specificity were calculated for optimal cut-off for $1^{\text {st }}$, $5^{\text {th }}, 10^{\text {th }}, 21^{\text {th }}$, and $24^{\text {th }}$ centiles of CCI. The inter-observer agreement was validated by interclass correlation coefficients (ICC).

\section{RESULTS}

A total of 149 participants were enrolled in this study. Among them, 12.08\% had PTB $(<37$ weeks $)$ and $87.92 \%$ had normal birth. Mean CCI for all subjects was $68.60 \%$, but was lower in PTB (48.9\%). The best cutoff for predicting PTB based on CCI was $59.46 \%$ with $99.18 \%$ sensitivity and $85 \%$ specificity. The AUC for CCI for prediction of PTB $<37$ weeks was 0.936 . There was no inter-observer difference in measurement of CCI and CL (ICC values for $\mathrm{CCI}$ and CL were 0.997 and 0.990 respectively).

\section{CONCLUSION}

In women with normal CL the CCI could predict PTB in the second trimester in low risk women. So, $\mathrm{CCI}$ is a valid predictor of PTB in low risk women.

Keywords: Pre term birth, cervical consistency index, low risk pregnancy
*Infertility \& Reproductive Health Research Center, Shahid Beheshti University of Medical sciences, Tehran, Iran

**Shohadaye Tajrish Hospital,

Shahid Beheshti University of Medical Sciences, Tehran, Iran

***Besat Hospital, AJA University, Tehran, Iran

\section{Correspondence:}

Fatemeh Salehi

Infertility \& Reproductive Health Research Center, Shahid Beheshti University of Medical sciences, Tehran, Iran

Email:dr.salehi1389@gmail.com

Date of first submission, May 20, 2018

Date of final revised submission, August 9, 2018

Date of acceptance, August 10, 2018

This open access article is distributed under a Creative Commons AttributionNon Commercial-Share Alike 4.0 International License

Cite this article as: Pooransari P, Salehi F, Afrakhteh M, et al. Cervical consistency index is a valid predictor of preterm birth in low-risk pregnant women. Univ Med 2018;37:150-8. doi: 10.18051/UnivMed.2018.v37.150158 


\section{INTRODUCTION}

Preterm birth (PTB) is defined as a delivery of an infant before 37 weeks of pregnancy. ${ }^{(1)}$ According to the WHO, each year about 15 million infants are born preterm and this number is rising every year, despite some progression in the medical field. ${ }^{(2)}$ The overall incidence of PTB ranges from 5 to $18 \%$ of infants born annually. ${ }^{(2)}$ So this complication becomes a major concern in gynecology and obstetrics. Preterm birth has been divided into three categories: extremely preterm (less than 28 weeks), very preterm (28-32 weeks) and moderate preterm (32-37 weeks). ${ }^{(2,3)}$ Preterm birth is responsible for neonatal morbidity and complications. These complications are due to the immature development of organs like the lungs, which is associated with respiratory distress syndrome (RDS), hence, the neonates may have cerebral palsy, vision problems, gastrointestinal complications, etc. ${ }^{(4,5)}$ The neonatal mortality rate has been decreasing in recent years, but PTB contributes to the death of 1.5 million infants annually. ${ }^{(6)}$ The mortality rate increases with decreasing gestational age. ${ }^{(7)}$ More than $75 \%$ of preterm births occurs during the $32^{\text {th }}$ to $36^{\text {th }}$ weeks of pregnancy. ${ }^{(8)}$ Some risk factors including; the maternal age at delivery, weight, the introduction of assisted reproductive technology (ART), have increased the incidence of PTB recently. ${ }^{(9)}$ Risk assessment like; history of previous abortion or PTB, Müllerian malformation, and cervical conization, multiple gestation, bleeding in the second trimester, and low pregnancy weight, are some of the predispositional factors for the occurrence of PTB in the current pregnancy, although their sensitivity is not reliable. ${ }^{(10)}$

In normal term, the cervix becomes soft but the uterus is closed until term, but in preterm birth the cervix shortens. ${ }^{(11)}$ Cervical length (CL), which is measured via transvaginal ultrasound (TVU), is a good predictor for PTB, especially in symptomatic women, ${ }^{(12)}$ although the accuracy of this technique is limited in asymptomatic women. ${ }^{(13)}$ It should be noted that about $50 \%$ of PTB occurs in asymptomatic women. ${ }^{(14)}$ Hence, despite determination of the CL index for the screening of PTB the rate has not decreased yet, especially in low-risk women. ${ }^{(15)}$

In the study of Parra-Saavedra et al. ${ }^{(16)}$ an ultrasonographic marker was investigated to measure cervix consistency throughout pregnancy quantitatively by transvaginal ultrasonography. Recently, a new technique based on measurement of the anteroposterior diameter of the cervix before and after cervical compression and calculating the cervical consistency index $(\mathrm{CCI})$, has been administered for prediction of PTB. ${ }^{(16)}$ It is shown that $\mathrm{CCI}$ is lower in women with preterm delivery and is also decreased in the third trimester of pregnancy. So, it could be a good indicator for PTB in low-risk women. ${ }^{(16)}$ Previous studies conducted on low risk women demonstrated that CCI was the better indicator of PTB in the second trimester, although women with $\mathrm{CL}$ under $25 \mathrm{~mm}$ were enrolled in previous studies where $\mathrm{CL}<25 \mathrm{~mm}$ is the predictor of PTB. In the current research, women with $\mathrm{CL}<25 \mathrm{~mm}$ were excluded, because medical intervention is needed in order to prevent complications such as PTB. So for the first time we evaluated normal pregnant women who were referred for routine screening and the association of CCI with the occurrence of PTB in this group was evaluated, and compared with the cervical length. ${ }^{(17)}$

Although CCI performed better than sonographic CL in predicting PTB, due to the still limited predictive capacity of these two measurements, other tools are needed to better identify women at increased risk of preterm delivery. ${ }^{(17)}$

Both CCI and CL measurements were significantly reduced in the high-risk compared with the low risk pregnancies. However, the diagnostic accuracy of both measurements was better in the low-risk population than in the highrisk population. ${ }^{(18)}$ 
The novelty of the study is that in the previous studies, in addition to healthy pregnant women with normal cervical length, pregnant women with reduced cervical length were studied. The present study did not include women with cervical length lower than 25 , who are at risk for early delivery. In addition, the cervical congestion index in healthy pregnant women with normal cervical length was studied. Because of the inclusion of normal cervical length and early delivery in the healthy pregnant women group, the present study is better for confirming the greater efficiency of CCI as compared to cervical length in predicting early delivery. The objective of this study was to determine the predictive value of cervical consistency index (CCI) to indicate the occurrence of PTB in mid trimester screening of low risk pregnant women.

\section{METHODS}

\section{Research design}

This was a prospective study conducted in Shohadye Tajrish Hospital, Tehran, Iran from April 2017 to March 2018.

\section{Research subjects}

The sample size was determined according to Cazanas et al. ${ }^{(19)}$ (effect size ${ }^{2}=0.082$ ) and the gestational age and CCI in linear multiple regression model for prediction of PTB were considered. The optimum sample size was 140 . Singleton pregnant women with low risk of preterm labor in the 14-28 weeks of pregnancy, including women who do not have the risk factors for preterm labor. The exclusion criteria were multiple pregnancies and use of drugs for the prevention of preterm labor (such as non-steroidal anti-inflammatory drugs, progesterones, magnesium sulfate, and calcium channel blockers), use of cerclage pessary, cervical length lower than $20 \mathrm{~mm}$ that requires the intervention to prevent preterm labor. Women with one of the preterm labor risk factors (previous preterm labor, loss of pregnancy due to cervical incompetency), cervical cone biopsy, and presence of molar anomalies in the reproductive system, were excluded from the study.

\section{Measurements}

Women with gestational ages in the range of 14-28 weeks underwent transvaginal sonography examination. The cervical length and anterioposterior diameter were measured twice during pregnancy and the CCI index was calculated. Gestational age was calculated according to reliable last menstrual period recall data or first trimester sonographic examination.

\section{Ultrasound examination}

The sonographic examination was carried out twice for each participant using GE VOLUSON E8 (GE Healthcare Ultrasound, Milwaukee, WI, USA) ultrasound machine which is equipped with a transvaginal probe. Cervical length, anterioposterior cervical diameter, gestational age, maternal weight, body mass index (BMI), age and parity were determined during the examination.

\section{Determination of cervical consistency index (CCI)}

In order to determine the CCI, the following steps were performed. Cervical length was measured according to the standard technique described in a previous study. ${ }^{(17)}$

The measurement of anterioposterior cervical diameter was carried out with an empty bladder and the cervical length was measured without any pressure. Then the midpoint of the line running along the longitudinal cervical axis was marked as $(\mathrm{C} / 2)$, then a line perpendicular to the longitudinal cervical axis through the point $\mathrm{C} / 2$ was drawn from the anterior lip to the posterior lip of the cervix. This length was designated the AP1 diameter. Then pressure was applied with the vaginal probe progressively until it reached the highest pressure at which there was no further shortening of the anterioposterior cervical diameter. Then the AP2 diameter was measured in a same way as described for AP1 
(Figure 1). To calculate the CCI the AP2 was divided by AP1 then multiplied by 100 :

$\mathrm{CCI}=(\mathrm{AP} 2 \mathrm{D}$ AP1 $) \times 100$

The measurement were performed twice and the lowest number was used for statistical analysis and inter-observer agreement. ${ }^{(16)}$ The patients were followed up to end of pregnancy and the outcome and date of delivery were recorded.

\section{Statistical analysis}

All data were analyzed with SPSS v.22 (IBM Copr, Armonk, NY). The gestational age in the second trimester (14-28 weeks) was evaluated to determine the ability of CCI to predict the PTB $(<37$ weeks). A multivariate logistic regression model for CCI and CL was constructed to assess the independent relation of each variable with PTB.

The relationship between quantitative variables was assessed with Pearson's correlation coefficient test. Distribution of CCI and $C L$ were analyzed in different percentiles according to gestational age at scan time.

Receiver operating characteristic (ROC) curves were drawn for CCI as predicting variable. Sensitivity, specificity, positive predictive value (PPV), negative predictive value (NPV), as well as positive and negative likelihood ratios (LR+ and LR-) were analyzed at $95 \%$ confidence interval to predict the PTB $<37$ weeks and the optimal cut-off point based on the ROC curves was calculated. All data obtained from the ROC curves demonstrate the diagnostic ability of CCI and CL to predict preterm birth. The area under the ROC curves (AUS) also was calculated. ${ }^{(18)}$

\section{Reliability of measurement}

The agreement between two measurements determined by different observers is called interobserver agreement. So, each participant was examined twice at one-hour interval by two observers. The differences were calculated using a Bland and Altman plot and intraclass correlation coefficient (ICC) ${ }^{(20)}$ Limits of agreement (mean differences \pm 1.96 ) (Standard Deviation*1.96) \pm mean) were calculated with the one-sample t-test and the systematic bias between two measurements was calculated at 95\% CI for mean difference.

\section{Ethical clearance}

Each participant signed an informed consent letter and the study was approved by the Ethics Committee of Shohadye Tajrish Infertility Research Center (under no. IR.SBMU.RETECH.REC.1397.306).

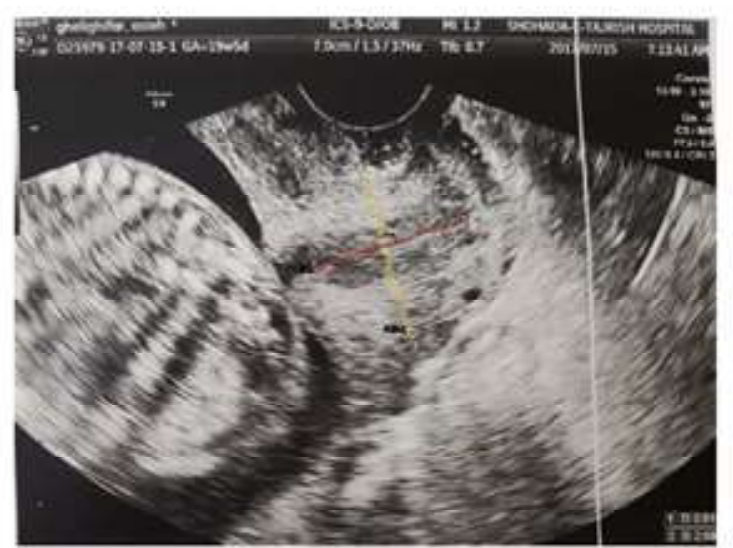

$\boldsymbol{A}$

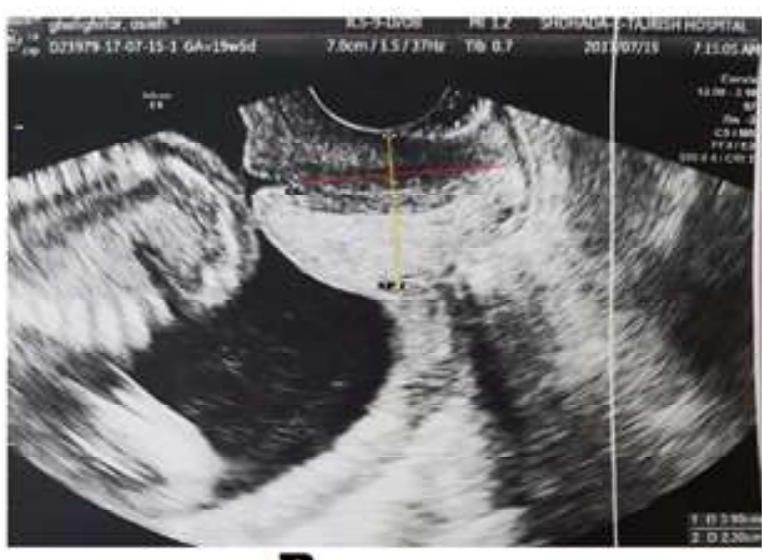

$\mathbf{B}$

Figure 1. Ultrasound image of cervix before (A) and after maximal compression with probe (B). The anterioposterior diameters (AP1, AP2) and cervical length (CL1, CL2) were measured in each position. The CCI index was determined from AP2/AP1*100 for each participant 
Table 1. Distribution of characteristics of patients enrolled in the study $(n=149)$

\begin{tabular}{lcccc}
\hline Characteristic & $\mathbf{n}=\mathbf{1 4 9}$ & $\begin{array}{c}\text { Normal birth } \\
(\mathbf{n = 1 3 1})\end{array}$ & $\begin{array}{c}\text { Preterm birth } \\
(\mathbf{n = 1 8})\end{array}$ & $\mathbf{p}$ \\
\hline Maternal age (years) & $28.93(16-44)$ & $29.4(17-44)$ & $25.2(16-36)$ & $>0.05$ \\
BMI before pregnancy & $25.06(20.2-35.56)$ & $25.1(20.2-35.56)$ & $24.17(20.3-29.62)$ & $>0.05$ \\
Weight gain (kg) & $14.49(4-30)$ & $14.70(4-30)$ & $12.94(6-22)$ & $>0.05$ \\
Gestational age at scan (weeks) & $19.42(14-27)$ & $19.29(14-27)$ & $20.3(17-28)$ & $>0.05$ \\
Gestational age at delivery (weeks) & $38(31-41)$ & $38.47(36-41)$ & $34.9(31-36)$ & 0.000 \\
Infant weight (g) & $3139(1870-4100)$ & $3218(2500-4100)$ & $2563(1870-3640)$ & 0.031 \\
Mode of delivery & & & & \\
Noninvasive delivery & $79(53.7 \%)$ & $67(51.1 \%)$ & $12(66 \%)$ & NS \\
CS & $69(46.3 \%)$ & $63(48.1 \%)$ & $6(33 \%)$ & NS \\
CCI (\%) & $68.60(34.86-93.02)$ & $71.32(50-93.02)$ & $48.9(34.8-64.61)$ & 0.000 \\
CL (mm) & $32.89(20.05-44.8)$ & $33.28(24.1-44.8)$ & $30.63(20.05-36.50)$ & $>0.05$ \\
\hline
\end{tabular}

BMI: body mass index; CS: cesarean; CCI; cervical consistency index; $\mathrm{Cl}$ : cervical length; *one case was excluded due to abortion in $17^{\text {th }}$ week of gestation

\section{RESULTS}

About 200 pregnant women, who were referred to Shohadye Tajrish Hospital, Tehran, Iran for routine examination, were enrolled in this study. A total of 51 women were excluded from the study due to several factors as follows. About 12 women were excluded due a maternal indication or a iatrogenic disorder before the $37^{\text {th }}$ week of pregnancy, such as fetal growth retardation, preeclampsia, non reassuring fetal assessment, 3 women with a history of multiple pregnancy, 8 women with a history of abortion ( $\leq 20$ weeks), and 28 women receiving progesterone for prevention of PTB. Participants with cervical length under $25 \mathrm{~mm}$ and having a non-horizontal cervix were also excluded from the study. All participants were followed up to delivery. About 149 pregnant women who were referred for a routine check-up in the second trimester were enrolled in this study. Among 149 pregnancies, 18 cases had PTB before 37 weeks
(12.08\%) and 131 cases had a normal birth $(87.92 \%)$. The prevalence of normal delivery was relatively higher than that of cesarean section $(53.7 \%$ vs. $46.3 \%)$. The characteristics of participants in the normal and preterm birth groups are summarized in Table 1.

There was no statistical difference in maternal age among women who had a normal birth and those with PTB(p>0.05). Sonography assessment results demonstrated that the mean cervical consistency index was $68.60 \%(71.32$ to $48.9 \%$ ) in which the percentage of CCI was lower in PTB (48.9\% vs. $71.32 \%)(\mathrm{p}=0.00)$ (Table 1).

In order to determine the best cut-off value of CCI, the ROC curves were used and the specificity, sensitivity, positive and negative predictive values and positive and negative likelihood ratio for best cut-off values to predict the PTB before 37 weeks were calculated. The best cut-off for predicting the PTB based on CCI was $59.46 \%$ (sensitivity $99.18 \%$, specificity

Table 2. Cervical consistency index cutoff based on ROC curve to predict PTB before 37 weeks

\begin{tabular}{ccccccc}
\hline Cut-off* & Sensitivity & Specificity & LR+ & LR- & PPV & NPV \\
\hline $47.75 \mathrm{p}(1)$ & $1(100 \%)$ & $50 \%$ & 2 & 0.5 & 21.56 & 100 \\
$54.6 \mathrm{p}(5)$ & $0.969(99.04 \%)$ & $65 \%$ & 2.768571 & 0.361197 & 27.99 & 99.79 \\
$57.32 \mathrm{p}(10)$ & $0.868(99.131 \%)$ & $70 \%$ & 5.526667 & 0.180941 & 31.22 & 99.82 \\
$59.46 \mathrm{p}(21)^{* *}$ & $0.829(99.18 \%)$ & $85 \%$ & 5.526667 & 0.180941 & 47.61 & 99.87 \\
$62.675 \mathrm{p}(24)$ & $0.775(99.23 \%)$ & $95 \%$ & 15.5 & 0.064516 & 73.6 & 99.88 \\
\hline
\end{tabular}

*Value lower than cutoff indicates high risk;**optimal cutoff based on ROC curve corresponds to $21^{\text {th }}$ centile LR + , positive likelihood ratio, LR-, negative likelihood ration, PPV, positive predictive value, NPV, negative predictive value, P1, $1^{\text {st }}$ centile; $\mathrm{p} 5,5^{\text {th }}$ centile; $\mathrm{p} 10,10^{\text {th }}$ centile; $\mathrm{p} 21,21^{\text {th }}$ centile; $\mathrm{p} 24,24^{\text {th }}$ centile 
$85 \%$ ), which according to the distribution of CCI in gestational ages at scan was located in percentile 21 (Table 2). The ROC curves for $\mathrm{CCI}$ are shown in Figure 2. AUC for CCI in relation to predicting the $\mathrm{PTB}$ before 37 weeks was 0.936 (95\%CI $0.891-0.982 ; \mathrm{p}=0.000)$. The specificity, sensitivity, positive and negative predictive values and positive and negative likelihood ratio for CCI and cut-offs of CCI in regard to predicting the.

To ensure the reliability of the CCI and CL measurements by one specialist, the ICC test was performed. The Bland-Altman plots (Figure 2) illustrate that there are no inter-observer differences in the measurement of CCI and CL.
The inter-observer ICC values for CCI and CL were 0.997 (95\%CI, 0.996-0.998) and 0.990 (95\%CI, 0.993-0.997) respectively. The interobserver agreement reliability of the CCI and CL (data was not shown).

\section{DISCUSSION}

The purpose of this study was to determine the diagnostic value of the cervical consistency index (CCI) in the prediction of preterm birth (PTB) (under 37 weeks) in low-risk pregnant women. Our results indicated that the CCI could be a reliable value for predicting PTB in a lowrisk population in the second trimester.

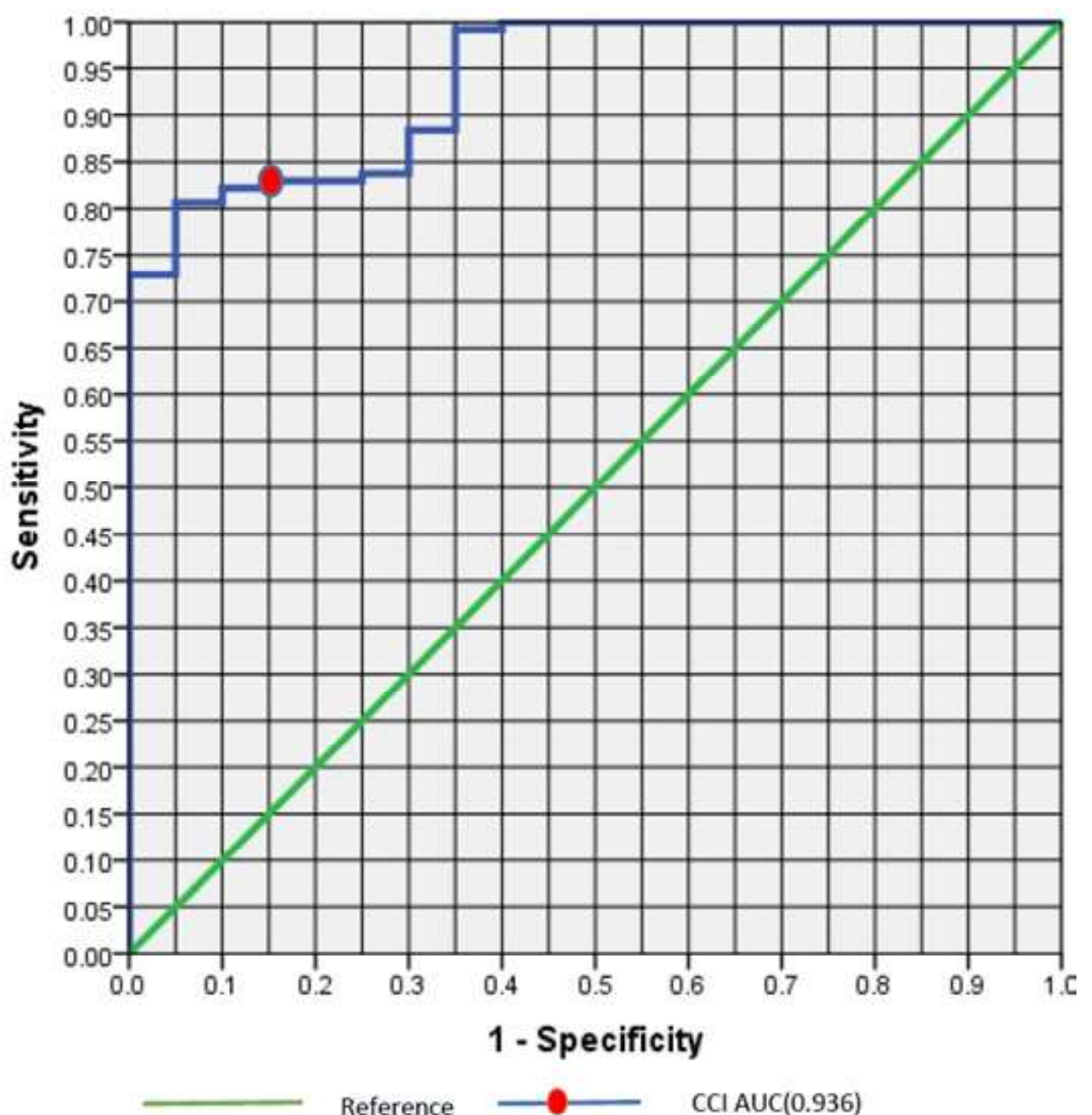

Figure 2. Receiver operating characteristics (ROC) curve for Cervical Consistency Index(CCI) for prediction of PTB before 37 weeks AUC: Area under ROC curve. Optimal cut-off based on ROC curve 
This was a first study in Iran to assess the CCI in low-risk pregnant women using transvaginal sonography. Evaluating the percentage of CCI in the second trimester (1428 weeks), demonstrated that low CCI in this period of pregnancy may lead to preterm labor (delivery before 37 weeks). ${ }^{(16)}$

In the current study, the PTB rate was $12.8 \%$ which was higher than that of a previous report from Iran,i.e. $5.6 \%{ }^{(21)}$ The PTB rate in the United States is about $12 \%$, which is similar to the current study. ${ }^{(22)}$ Preterm birth (PTB) is one of the major causes of neonatal mortality in the population. ${ }^{(23)}$ According to the WHO the PTB rate ranges from 5 to $18 \%$ and about $5-7 \%$ of infants die due to PTB in developed countries. ${ }^{(2)}$ This indicates that the PTB rate is higher in developing countries and because the current study was just about one hospital in Tehran, some socio-economic differences may also have an effect on the prevalence of PTB. ${ }^{(24)}$

In our study, in order to predict the PTB risk, the cervical length was determined. ${ }^{(18)}$ However, a CL of less than $25 \mathrm{~mm}$ indicates the need for medical intervention in order to prevent the complications of PTB. ${ }^{(10)}$ In low-risk women with a CL higher than $25 \mathrm{~mm}$ no medical intervention is necessary, but as demonstrated in this study the rate of PTB in pregnant women with CL above $25 \mathrm{~mm}$ was noticeably high (12\%). So it is clear that CL could not be a reliable factor for predicting PTB. According to a previous study, changes in cervical length may be a late outcome and softness of the cervix occurs before shortening, and water concentration during the ripening process may not cause any clear symptoms and could not be detected in the first trimester. ${ }^{(25)}$

The assessment of cervical consistency by pelvic examination did not have any clinical value for predicting PTB. For the first time, cervical consistency was measured by transvaginal sonography. The CCI index in our study was lower in women with preterm delivery than in those with term delivery, as was also demonstrated in other studies. The low cervical consistency index indicates weakness of the cervix and is directly related to preterm birth. ${ }^{(26)}$

In order to determine the predictive value of CCI for detection of PTB, the cutoff value is needed. According to our study, the best cutoff for predicting PTB before 37 weeks was $59.46 \%$ with $85 \%$ specificity, $99.18 \%$ sensitivity, $L R+5.52$, and LR- 0.18 , which is connected with percentile 21 of the gestational week. The study by Baños et al. ${ }^{(18)}$ found the best cutoff to predict the PTB before 37 weeks in percentile 20 (corresponding to CCI $64.6 \%$, sensitivity $72 \%$, specificity $61.2 \%$ LR+1.9 and LR- 0.4).

In order to confirm the inter-observer differences in the measurement of CCI and CL, the ICC value was calculated and was 0.99 in both measurements. So the measures obtained were reliable and sufficient for clinical use in both CCI and CL.

The main limitation of our study is the limited number of PTB $(n=18)$ cases, due to the low risk population selection of the study. However, in the current study, in which only low risk people were selected and women with CL under $25 \mathrm{~mm}$ were excluded from the study, a good relation between $\mathrm{CCI}$ and PTB in the low risk population has been obtained, so indicating the validity of the current study.

This study was conducted in one hospital, but in order to confirm the reliability of our results, it is better to repeat it in other centers with large populations. Finally, due to lack of persons with expertise for measuring the anterioposterior diameter of cervix and determining the CCI, we did not have inter-observer agreement. With regard to the increase of the expert people in the measurement of the cervical compression index, it is better that the present study be performed in a larger and multi-centre study by a number of people, allowing conclusions to be made on interobserver reliability.

\section{CONCLUSION}

Determination of CCI in asymptomatic pregnant women could prevent occurrence of 
PTB and medical implication following complications in the newborn, hence CCI should be calculated in routine sonography examination.

\section{ACKNOWLEDGEMENT}

We thank our colleagues from Shohadaye Tajrish Hospital for their assistance with sonograpghic examination and we are also immensely grateful to Dr Hanieh Rezaee for her support on manuscript preparation.

\section{CONFLICT OF INTEREST}

All authors declare that they do not have any conflict of interest.

\section{CONTRIBUTORS}

PP contributed to the basic concept and design of the study. FS and MA contributed to writing the manuscript and performing the experiment. MM and SSG contributed to sample preparation and data collection. OGS contributed to the statistical analysis. All authors read and approved the final manuscript.

\section{REFERENCES}

1. Kemp MW. Preterm birth, intrauterine infection, and fetal inflammation. Front Immunol 2014;5:574. doi: 10.3389/fimmu.2014.00574.

2. World Health Organization. Preterm birth. Geneva: World Health Organization;2017.

3. Blencowe H, Cousens S, Oestergaard MZ, et al. National, regional, and worldwide estimates of preterm birth rates in the year 2010 with time trends since 1990 for selected countries: a systematic analysis and implications. Lancet 2012; 379:2162-72. doi: 10.1016/S0140-6736(12)60820-4.

4. Saigal S, Doyle LW. An overview of mortality and sequelae of preterm birth from infancy to adulthood. Lancet 2008;371:261-9. doi: 10.1016/ S0140-6736(08)60136-1.

5. Hafstrom M, Kallen K, Serenius F, et al. Cerebral Palsy in Extremely Preterm Infants. Pediatrics. 2018;141:e20171433. doi: 10.1542/peds.2017-1433.

6. Lawson MJ. The role of inflammation in the pathogenesis of preterm birth [thesis].Cincinnati, Ohio: University of Cincinnati; 2017.
7. Gargari SS, Kashanian M, Zendedel H, et al. Survival and risk factors of extremely preterm infants ( $<28$ weeks) in the three Iranian Hospitals. Acta Medica Iranica 2018;56:181-8.

8. Simmons LE, Rubens CE, Darmstadt GL, et al. Preventing preterm birth and neonatal mortality: exploring the epidemiology, causes, and interventions. Semin Perinatol 2010;34:408-15.

9. Tamura N, Hanaoka T, Ito K, et al. Different risk factors for very low birth weight, term-small-forgestational-age, or preterm birth in Japan. Int J Environ Res Public Health 2018;15:369. doi: 10.3390/ijerph15020369.

10. Koullali B, Oudijk MA, Nijman TA, et al. Risk assessment and management to prevent preterm birth. Semin Fetal Neonatal Med 2016;21:80-8. doi: 10.1016/j.siny.2016.01.005.

11. Myers KM, Feltovich H, Mazza E, et al. The mechanical role of the cervix in pregnancy. $\mathrm{J}$ Biomech 2015;48:1511-23. doi: 10.1016/j.jbiomech. 2015.02.065.

12. Romero R, Nicolaides K, Conde Agudelo A, et al. Vaginal progesterone decreases preterm birth $\leq 34$ weeks of gestation in women with a singleton pregnancy and a short cervix: an updated meta analysis including data from the OPPTIMUM study. Ultrasound Obstet Gynecol 2016;48:30817. doi: 10.1002/uog.15953.

13. Facco FL, Simhan HN. Short ultrasonographic cervical length in women with low-risk obstetric history. Obstet Gynecol 2013;122:858-62. doi: 10.1097/AOG.0b013e3182a2dccd.

14. Dekker GA, Lee S Y, North RA, et al. Risk factors for preterm birth in an international prospective cohort of nulliparous women. PLoS ONE 2012;7: e39154. doi: 10.1371/journal.pone.0039154.e39154.

15. Iams JD, Romero R, Culhane JF, et al. Primary, secondary, and tertiary interventions to reduce the morbidity and mortality of preterm birth. Lancet 2008;371:164-75. doi: 10.1016/S01406736(08)60108-7.

16. Parra-Saavedra M, Gomez L, Barrero A, et al. Cervical consistency index: a new concept in uterine cervix evaluation. Ultrasound Obstet Gynecol 2011 ;38:44-51. doi: 10.1002/uog.9010.

17. Banos N, Julia C, Lorente N, et al. Mid-trimester cervical consistency index and cervical length to predict spontaneous preterm birth in a high-risk population. Am J Perinatol Rep 2018;8:e43-50. https://doi.org/10.1055/s-0038-1636993.

18. Banos N, Murillo-Bravo C, Julia C, et al. Midtrimester sonographic cervical consistency index to predict spontaneous preterm birth in a lowrisk population. Ultrasound Obstet Gynecol 2017; 51:629-36. doi: 10.1002/uog.17482. 
19. Cazanas A, de San Miguel A, Parra E. Estimating sample size for usability testing. Enfoque UTE 2017;7:172-85.

20. Stolarova M, WolfC, Rinker T, et al. How to assess and compare inter-rater reliability,agreement and correlation of ratings: an exemplary analysis of mother-father and parent-teacher expressive vocabulary rating pairs. Front Physiolog 2014;5:113. doi: $10.3389 /$ fpsyg.2014.00509.

21. Amini P, Maroufizadeh S, Samani RO, et al. Prevalence and determinants of preterm birth in Tehran, Iran: a comparison between logistic regression and decision tree methods. Osong Public Health Res Perspect 2017;8:195-200. doi: 10.24171/j.phrp.2017.8.3.06.

22. Martin J, Hamilton B, Ventura S, et al. Births: final data for 2010. Natl Vital Stat Rep 2012;61:1-72.
23. Liu L, Oza S, Hogan D, et al. Global, regional, and national causes of child mortality in 2000-13, with projections to inform post-2015 priorities: an updated systematic analysis. Lancet 2015;385: 430-40. doi: 10.1016/S0140-6736(14) 61698-6.

24. Goldenberg RL, Culhane JF, Iams JD, et al. Epidemiology and causes of preterm birth. Lancet 2008;371:75-84. doi: 10.1016/S0140-6736(08)600744.

25. Donoghue D, Lincoln D, Morgan G, et al. Influences on the degree of preterm birth in New South Wales. Aust N Z J Public Health 2013;37: 562-7.

26. Myers KM, Feltovich H, Mazzad E, et al. The mechanical role of the cervix in pregnancy. $\mathrm{J}$ Biomech 2015;48:1511-23. doi: 10.1016/j.jbiomech. 2015.02.065. 